\title{
PACS in 2018: An Autopsy
}

\section{Don Dennison}

Published online: 19 December 2013

(C) Society for Imaging Informatics in Medicine 2013

It's 2018 and a lot has changed for medical imaging in the past 5 years - most notably, the death of Picture Archiving and Communication System (PACS). Let's take a look back at how it happened (Fig. 1).

\section{4}

For years leading up to 2014, there was lots of talk about enterprise imaging and ordering. With the shift from volumebased reimbursement to quality measures taking hold, attention turns to expanding image management services beyond traditional diagnostic departments and to improving services to referring physicians.

\section{Reliable Enterprise Image Capture to VNA Becomes Common}

Lots of solutions claimed the ability to capture clinical images, but most lacked the basic ability to capture the desired metadata for the image, to make the images effectively interoperable, or to integrate the capture process into the clinical routines without significant disruption. In 2014, this started to change. The advent of Web technologies, such as mobile device cameras and accessories and Representational State Transfer (REST) [1] Web services, allowed a new image and data capture platform to emerge. Images were easy to capture, patient and location identification methods were automated and improved, and the creation of standard-based interoperable images stored to the Vendor Neutral Archive (VNA) became the norm. Zero-install [2] Enterprise Viewers, connected to the VNA and embedded in the Electronic Medical

D. Dennison $(\bowtie)$

Don K Dennison Solutions Inc, Waterloo, ON, Canada

e-mail: don@dondennison.com
Record (EMR) and Health Information Exchanges (HIE), provided a single, longitudinal view of diagnostic and clinical images and information for patients.

\section{Enterprise Order Management, Scheduling and CPOE} Rapidly Adopted

Historic physician referral patterns were in upheaval. Led by the shift to Accountable Care Organizations (ACO) and patient steerage (payers directing patients to lower cost imaging providers), diagnostic imaging service providers responded by improving the quality of service they provided through the use of some new tools. To get physicians to refer patients to them, providers had to make it simple and easy for physicians to place orders, schedule appointments, and get results. Enterprise Order Management provided a real-time ability to place orders for exams, regardless of the diagnostic imaging department, and even schedule them, while the patient was still in their office, often doing this from their tablet device. The ordering application prompted the referring physician to ensure that they ordered the most medically appropriate imaging exam based on evidence [3]. Initial patient information, such as pregnancy, allergies, and pace maker status, was pulled automatically from the EMR and HIE to assist in the order placing logic.

Enterprise Viewers Provide Essential Advanced Visualization

Enterprise Viewers emerged in the late 2000s but significantly grew in capability by 2014 . Not only had the navigation tools improved but also mobile access was common. Web browsers had gotten faster and more stable than ever before. However, the big improvement in 2014 was in their advanced visualization capabilities. Comprehensive and interactive Maximum Intensity Projection (MIP), Multi-Planar Reconstruction (MPR), and 3D became generally available. New middleware 


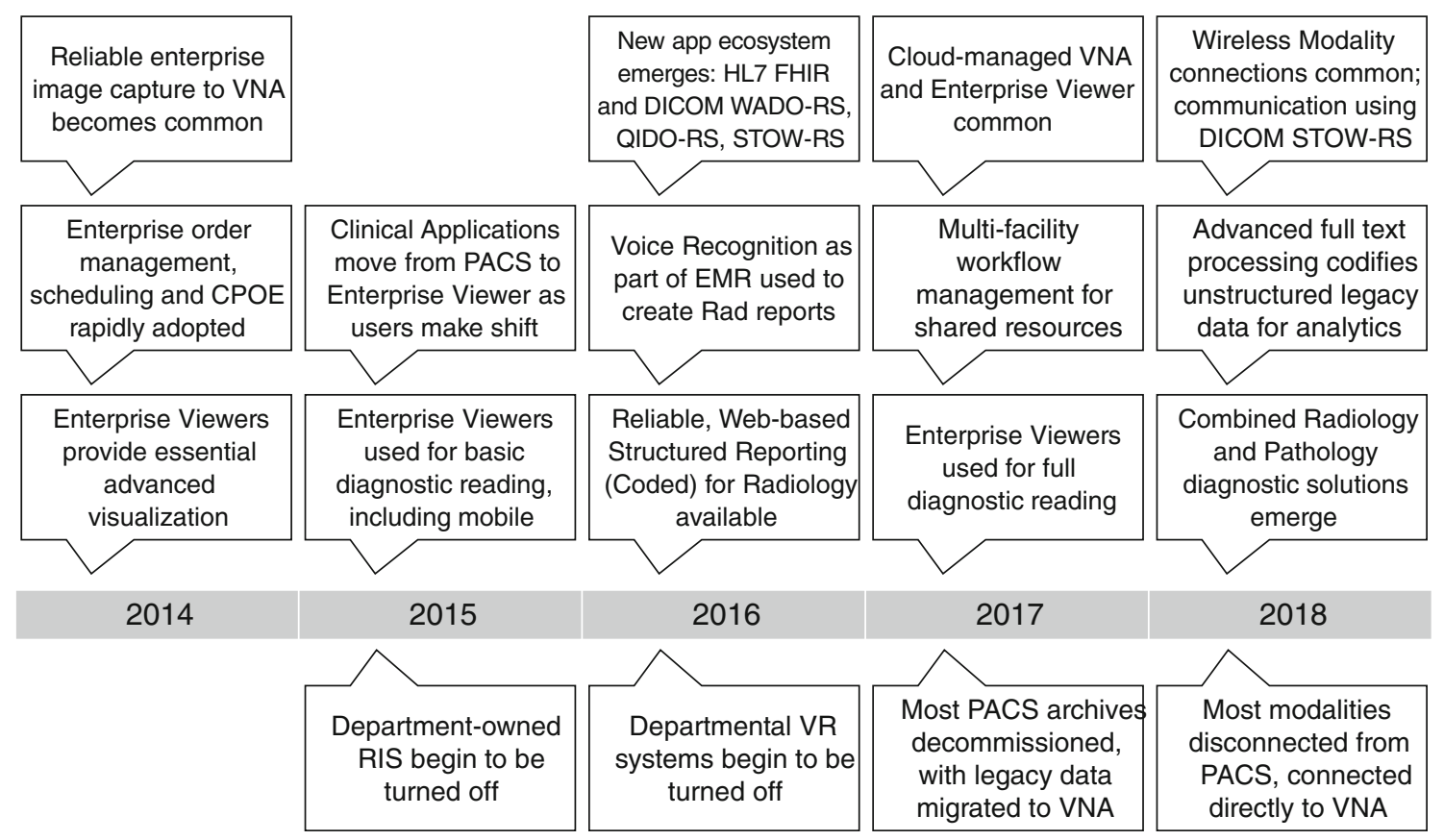

Fig. 1 A time line of innovations leading to the death of PACS

Application Programming Interfaces (APIs) and proxy capabilities began to emerge, allowing data from multiple sources - such as departmental PACS and VNA systems at different organizations - to be displayed, analyzed, and compared in a single, familiar Web interface.

\section{5}

The impact of the shift of users from PACS clients to Enterprise Viewers reached the tipping point in 2015. By then, there were far more users accessing the Enterprise Viewer-from within the EMR and HIE as well as directly-instead of the PACS. The convenience of the Enterprise Viewer even began to appeal to the diagnostic users for routine use.

\section{Clinical Applications Move from PACS to Enterprise Viewer} as Users Make Shift

Previously, if a clinician needed specialized analytic, visualization, or navigation tools, they would use a clinical application connected to the PACS or one to which the PACS was forwarding images. The tools - though expensive-mostly worked, but having to $\log$ in to a separate system, dealing with disruptive upgrades that broke things and lack of mobile access left users wanting. As they stopped using PACS to view images, they demanded that the clinical tools be made available on the Enterprise Viewer Web applications. As this new generation of clinical tools needed to be zero-install Web applications too, new vendors emerged, while others adapted. Usability became a significant differentiator. Integration into the Enterprise Viewers proved to be simpler to maintain, and security was improved. Support and maintenance agreements for legacy clinical tools were not renewed, and the applications removed.

Enterprise Viewers Used for Basic Diagnostic Reading, Including Mobile

With the majority of users now on the Enterprise Viewer platform, enjoying accessibility and collaboration tools they never had on the PACS, the primary diagnostic users found themselves behind the times. And perhaps worse, even less connected to the patient's circle of care. Radiologists, cardiologists, and other departmental staff used their significant imaging and informatics knowledge to help their facilities choose Enterprise Viewers that could replace their PACS. This was not as easy as it seemed, as the PACS had deep roots, but the evolution was started. Diagnostic users began interacting and consulting with referring physicians and clinicians, reading studies from their tablet device — wirelessly displaying[4] information on high-resolution monitors at stations conveniently located nearby, as needed.

\section{Department-Owned RIS Begin to be Turned off}

By 2015, many facilities had shifted to using the Radiology Information System (RIS) that was supplied as part of their new EMR. Operations were often disrupted and the change was painful; however, the economics of radiology made the change inevitable. As the EMR-provided RIS slowly began to improve with new versions (driven by feedback from users 
that shifted from a more mature product) and flexible customizations, operations became more efficient and reliable. Departmental RIS support and maintenance contracts were not renewed, and RIS applications were gradually turned off. Smaller facilities began trying pure Cloud-based applications to manage their resources, workflow, operations, and department finances-Customer Relationship Management (CRM) modules offered new capability to manage referring physicians. Some remnants, such as the reporting module, remained standalone applications for a while - but this would not last for long.

\section{6}

Long discussed, structured reporting finally came to radiology in 2016 and in a big way. Riding the wave of innovation driven by new Web-based standards, new tools quickly emerged. They offered unsurpassed interoperability and intelligence.

\section{New App Ecosystem Emerges: HL7 FHIR and DICOM WADO-RS, QIDO-RS, STOW-RS}

Starting back in 2012, new standards for healthcare - including Health Level 7 (HL7) Fast Health Interoperability Resources (FHIR) and Digital Imaging and Communications in Medicine (DICOM) Web Access to DICOM Persistent Objects by RESTful Services (WADO-RS), Query based on ID for DICOM Objects by RESTful Services (QIDO-RS), and Store Over the Web by RESTful Services (STOW-RS)[5] which use the latest Web API designs and protocols were being defined. They got traction in 2013 and 2014, but in 2015 and 2016, they began to appear in several vendors' products. Some were extensions to existing products from established vendors - including image management, EMR, and HIE providers - while others were provided as a middleware platform from innovative start-ups. The new age of interoperability had arrived. Images and information were available to a whole new class of application, with better security and privacy protection than ever before. Real-time and online collaboration changed the way physicians interacted with each other, and patients.

\section{Voice Recognition as Part of EMR Used to Create Rad Reports}

With the EMR came data entry-in other words, typing. Advances in voice recognition and audio input hardware provided new opportunities for capturing information without typing. Mobile devices, carried by physicians and other healthcare workers, led the way. Innovations in predictive text, hardened by the mobile input industry, and mature medical ontologies led to unprecedented productivity enhancements, along with automated methods for coding data. Browsers included APIs to turn real-time audio capture into the text input needed for these processes, making application development rapid and affordable.

Reliable, Web-based Structured Reporting (Coded) for Radiology Available

By 2016, the technology had evolved to a point where radiology report authoring applications could create structured, coded reports that were available immediately upon finalization (using biometrics, such as voice print signature and facial recognition, for signing them in real-time). Online libraries and APIs provided real-time discovery and access to the best structured report template for the imaging procedure. Access to key patient and order information and the distribution of the finalized report was shifting from traditional HL7 methods to using HL7 FHIR, and applications were starting to be offered in the Cloud. Functional (e.g., measurements) and technical (e.g., radiation dose) information is extracted from image management systems and included in reports, using DICOM QIDO-RS and WADO-RS, as needed.

\section{Departmental VR Systems Begin to be Turned off}

With evidence that the technology was ready and research showing the benefits of structured, coded radiology reports, reporting applications were shut off.

\section{7}

As user-facing applications-like image viewing, report creation, and results access - had shifted to new platforms from legacy RIS and PACS to Enterprise Viewers, EMR modules, and Web-based information systems, the time for facilities that had made the change in order to expand services and grow had arrived.

\section{Cloud-managed VNA and Enterprise Viewer Common}

Well before 2017, the use of VNA for multisystem imaging information consolidation, enterprise imaging management, and cross enterprise sharing had become commonplace. But, it was around this time that the use of these systems in the Cloud went from novelty to routine. Standards, such as DICOM STOW-RS, allowed secure and reliable communication between local enterprise systems and the VNA in the cloud without relying on a LAN or VPN connection. The Enterprise Viewer in the Cloud, connected to one or more VNA, provided secure access to images for EMR and HIE users. As with all Cloud applications, scaling out processing capability and 
storage capacity on demand is now elastic and automatic. System monitoring and platform maintenance is handled by the Cloud provider, allowing providers to focus on the data and its meaning.

\section{Multi-facility Workflow Management for Shared Resources}

As organizations consolidated or formed ACOs, or simply extended their affiliations, the need to share resources reached a peak. While Enterprise Ordering and Scheduling has already provided productivity enhancements for booking imaging exams when and where it was best for the patient, the need to balance workloads and to get access to specialists for complex cases finally took center stage. New applications emerged which balanced the reading work among the best available and qualified radiologists and other imaging specialties. They managed credentialing and licensing across the involved organizations. Building off the middleware APIs provided by the VNA, information systems, and Enterprise Viewer, a new model of work task optimization was born.

\section{Enterprise Viewers Used for Full Diagnostic Reading}

By 2017, even die hard PACS users were moving toward using the Enterprise Viewer for primary diagnosis. Enterprise Viewers were performing well on high-resolution monitors, in multiple monitor workstation configurations as well as on mobile devices. Updates to legacy PACS applications were dwindling, and IT staff was eager to cease providing desktop support, so many PACS were removed from operations in this year.

Most PACS Archives Decommissioned, with Legacy Data Migrated to VNA

For years, the VNA applications had managed newly acquired imaging data, but migration of legacy data can take years, especially for high-volume facilities that went digital early. With the last of the data migrated, and several years of proven VNA operation, both hosted locally and in the Cloud, the remaining PACS archives are shut down.

\section{8}

Now, only highly efficient healthcare providers have survived. They have done so through the investment in the right IT, as part of an overall multiphase plan, and a clear focus on change management. Successful leaders have used data and metrics to guide both operational and quality outcome improvements. They have overhauled their environment from a collection of domain-specific equipment to a powerful platform of secure, purposeful services with which the craft of healthcare delivery can be transformed.

Wireless Modality Connections Common, Communication Using DICOM STOW-RS

Wireless networks are reliable and ubiquitous inside and outside the hospital. Full modalities, as well as new handheld editions for some diagnostic exams, can communicate without being tethered to a cable. Clinical image capture is seamless, with transmission from sophisticated cameras to mobile devices for proper informatics and then on to the VNA. Communication of information and images over standard protocols, such as DICOM QIDO-RS, WADO-RS, and STOW-RS, are more common now than legacy DICOM methods.

Advanced Full Text Processing Codifies Unstructured Legacy Data for Analytics

Structured and coded information is much more commonplace, but there are still lots of legacy information in documents and paragraph form that are disconnected from the rest of the patient's information. Advances in full text processing applications, integrated with coded ontologies through RESTbased Web APIs, securely crawl through volumes of stored text and updates the structured data indices.

\section{Combined Radiology and Pathology Diagnostic Solutions Emerge}

The technology to convert the practice of pathology from reading slides through a microscope to a digital solution emerged several years ago. After a generation of mostly proprietary systems, and failed attempts to combine with radiology PACS, the right integrated solution - that pathologists accepted - became available this year. As the two diagnostic practices of pathology and radiology blend into a set of common integrated services, the information is also integrated as part of Web-based imaging and information platform, all accessible right in the EMR.

Most Modalities Disconnected from PACS, Connected Directly to VNA

With all the changes implemented - with legacy image data migrated to the VNA, the RIS replaced by services in the EMR, the PACS client by the Enterprise Viewer, and the reading workflow tasks managed across teams by a shared multi-facility application - the last bastion of PACS is the interface to the Modalities. While VNA have had DICOM interfaces to receive images since the beginning, PACS have long provided specific interfaces, such as DICOM Modality Worklist (DMWL) and Modality Performed Procedure Step 
2013

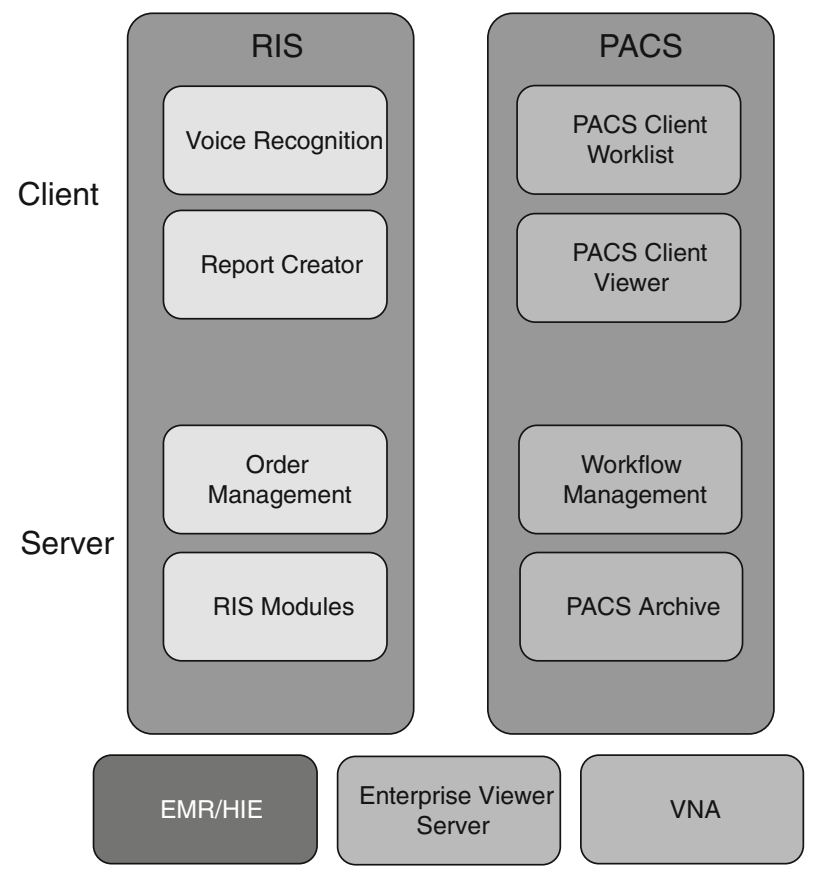

2018

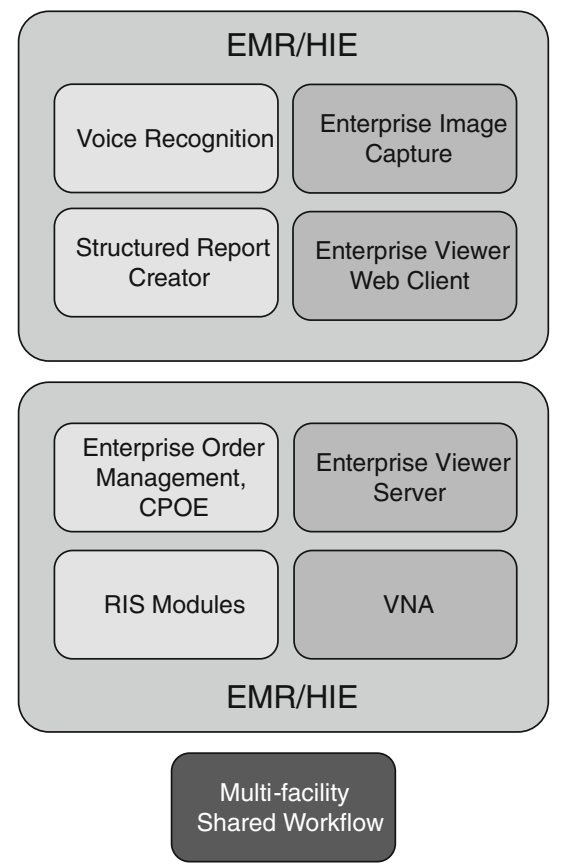

Fig. 2 Evolution of systems from 2013 to 2018

(MPPS) management, to manage the acquisition workflow process with modalities. As some modalities lacked effective interfaces, the PACS has had to compensate. By now, VNA vendors have not only provided the type of adaptive interfaces needed to support direct modality integration but are also providing new Web-based interfaces for modern acquisition modalities. Modalities are being disconnected from the PACS and integrated directly with the VNA. PACS is now dead.

\section{System Evolution}

Please see Fig. 2

\section{Author Notes, Discussion}

While this paper explores some major changes to the current state-of-the-art, the industry has been through a similar level of change and come out the other side improved. Consider how people in the provider and vendor communities felt about going digital with PACS and RIS when all they knew was film and paper. I predict that the shift to the new reality described here will happen faster than that change, as much of the technology is available and proven - it just needs to be adapted for healthcare.

\section{References}

1. Fielding RT, Architectural Styles and the Design of Network-based Software Architectures, 2000; Chapter 5 Representational State Transfer (REST) Available at http://www.ics.uci.edu/ fielding/pubs/ dissertation/rest_arch_style.htm and Chapter 6 Experience and Evaluation Available at http://www.ics.uci.edu/ fielding/pubs/ dissertation/evaluation.htm

2. Applications with a pure Web client (e.g. using HTML5) and no software installed on the client/device

3. Healthcare IT, CPOE definition. Available at http://www. healthcareitnews.com/directory/computerized-physician-order-entrycpoe. Accessed 14 October 2013.

4. Over WiDi (or similar method); Intel. Available at http://www.intel. com/go/widi. Accessed 14 October 2013.

5. DICOM Working Group 27 (WG-27) defines the WADO-RS, QIDORS and STOW-RS standards; WG-27 Minutes: http://medical.nema. org/DICOM/minutes/WG-27/ 